\title{
Regulating DCs in Innate Immune Response and Infection by Cytoskeletal Proteins
}

Wei Lin ${ }^{1^{*}}$ and Xin Wang ${ }^{2^{*}}$

${ }^{1}$ Department of Microbiology, Institute of Basic Medicine, Shandong Academy of Medical Sciences, Jinan, Shandong 250032, PR China

2Department of Clinical Laboratory, Qilu Hospital, Shandong University, Jinan, Shandong 250012, PR China

"Corresponding authors: Wei Lin Ph.D., Department of Microbiology, Shandong Academy of Medical Sciences, 18877 Jingshi Road, Jinan, Shandong 250030, PR China, Tel: 8615098926353; E-mail: linw1978@163.com

Xin Wang Ph.D., Department of Clinical Laboratory, Qilu Hospital, Shandong University, Jinan, Shandong Province 250012, PR China, E-mail: wx198116@163.com

Received date: April 23, 2017; Accepted date: May 16, 2017; Published date: May 22, 2017

Copyright: (c) 2017 Lin W, et al. This is an open-access article distributed under the terms of the Creative Commons Attribution License, which permits unrestricted use, distribution, and reproduction in any medium, provided the original author and source are credited.

\begin{abstract}
Dendritic cells (DCs) are important antigen-presenting cells of innate immune system. Their main function is to take up antigen and present it to T-cells to active adaptive immune response. The cytoskeletal proteins of the DCs not only maintain the morphology of DCs, but also are associated with the maturation and function of DCs. Additionally, rearrangement of cytoskeletal proteins is associated with viral infection in DCs. Regulating the specific cytoskeletal proteins of DCs would be a potential therapeutic strategy for preventing viral infection. Thus, the role of cytoskeletal proteins of DCs in immune response and viral infections were reviewed.
\end{abstract}

Keywords: Dendritic cells; Immune response; Cytoskeletal proteins; F-actin

\section{Introduction}

Dendritic cells (DCs) are important antigen-presenting cells of innate immune system. They play a key role in the initiation of immunity by recognizing and integrating signals from a wide range of pathogens. DCs are distributed throughout the body and their main function is the uptake of self and/or foreign antigens. During this process, immature DCs become mature and induce drastic changes in the gene expression, protein synthesis, and surface transport. These processes allow DCs to gain the capability to migrate and uptake antigen [1]. Following antigen uptake, DCs migrate to the T-cell areas of the lymph nodes [2] in order to present antigen-derived peptides in the context of the major histocompatibility complex (MHC) molecules to induce antigen-specific T-cell activation [1]. The majority of functional hallmarks of DCs are tightly regulated by the cytoskeletal proteins, which promote the cell polarization and intracellular redistribution of proteins and organelles to aid the migration of DCs and their activation [3]. Recently, certain studies reported that the cytoskeletal proteins of DCs participate in the immune response and viral infection by regulating the function of DCs. Herein, we reviewed the role of cytoskeletal proteins of DCs in the immune response and the viral infection.

\section{The F-actin of DCs and Immune Response}

Cytoskeletal proteins form a complex network that is distributed throughout the cytoplasm, from the nucleus to the plasma membrane. They are classified into three main proteins including microfilaments, intermediate filaments and microtubules. Microfilaments are composed of linear polymers of G-actin proteins namely, myosin and F-actin filaments, and are controlled by the Rho family of small GTPbinding proteins, such as Rho, Rac, Cdc42, WASP/N-WASP/WAVE, the ARP2/3 complex, and the formin family of actin-binding proteins [4-6]. The function of the microfilaments is associated with the maintenance of the cell-shape, cell migration, and the redistribution of molecular signalling. Beside microfilaments, intermediate filaments are also responsible for the maintenance of cell-shape and organization of internal tridimensional structure of the cell. And they participate in certain cell-cell adhesion and cell-matrix junctions. Microtubules comprise thirteen protofilaments. They possess a dynamic behaviour via their binding to GTP for polymerization and their organization by the centrosome. The role of cytoskeletal proteins in the cells can be summarized as follows (1) These proteins are responsible for cell shape and form specialized structures, such as flagella, cilia, lamellipodia and podosomes. (2) They are required for cell-cell contact and are associated with extracellular connective tissue and other cells in order to stabilize certain tissues [7]. The cytoskeleton can also actively contract, thereby deforming the cell and the cell environment and allowing cells to migrate $[3,8]$. (3) Moreover, cytoskeletal proteins are involved in a multitude of cell signalling pathways $[9,10]$ cytokinesis secretion $[3,11]$ and intracellular transportation.

The cytoskeletal proteins of DCs not only maintain the morphology of DCs, but also affect DC maturation, which determines the immune activation or the immune tolerance. Immature DCs are induced to be mature to express high levels of the markers CD80, CD86 and CD83 on their surface. And their morphology change to stretch dendritic spines, which are suggested to play an important role in synaptic plasticity. Dendritic spines contain a high concentration of actin $[12,13]$, which plays a central role in supporting cell motility, morphological changes, antigen uptake and immunological synapse formation between DCs and T-cells. The polymerization of actin filaments (F-actin) is most likely involved in the spinal shape changes in order to sustain the function and biological state of the dendritic cells (Figure 1A). If the arrangement of F-actin is disrupted, DC became round with short dendrites, and lost the ability to become mature [14]. 
A
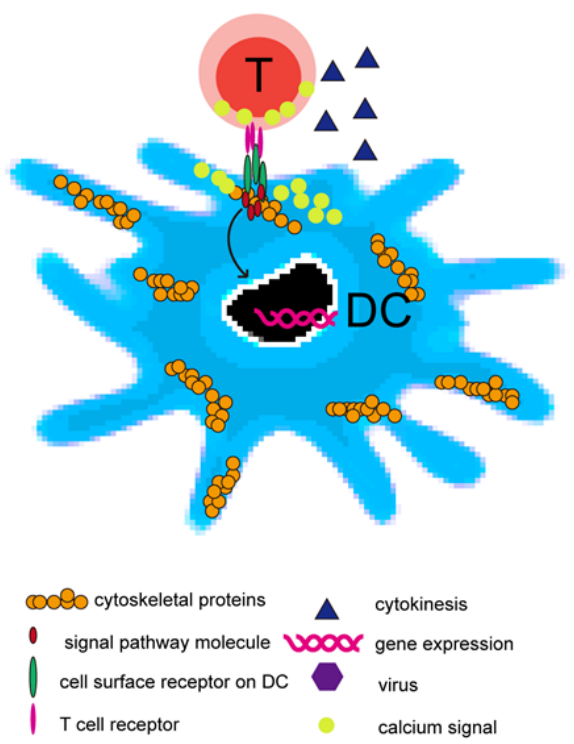

B

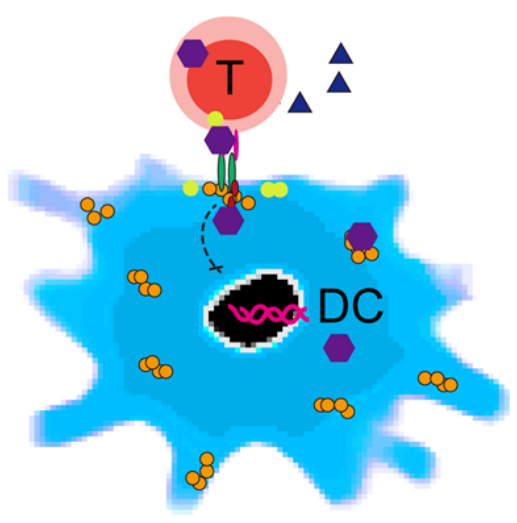

Figure 1: The diagram of cytoskeletal proteins in DCs. (A) Cytoskeletal proteins distribute throughout the cytoplasm of DCs. They maintain the shapes of DCs to form the dendrites. Cytoskeletal proteins are helpful for surface molecules accumulation, signalling pathway activation, and cytokines secretion. And cytoskeletal proteins arrangements promote the contact formation between T-cell and DC cells to induce the calcium release in T-cells. (B) Virus recognize the cell surface molecules to enter into DCs and interrupt the aggregation of cytoskeletal proteins, resulting in morphological change of DCs and the damage of surface molecules accumulation, signalling pathway activation and cytokines secretion of DCs. And virus could be transported into T-cells through the contact between DC and T-cells.

\section{Antigen uptake requires F-actin}

The function of DCs requires actin organization. During the process of uptake antigen, F-actin in DCs plays a role in the expression and accumulation of cell surface molecules that are required to recognize antigens and facilitate their endocytosis. DCs are important APCs that recognize antigens and facilitate their uptake. During this process, actin polymerization generates force in order to affect the internalization of plasma membrane vesicles, which contain the antigens. A large amount of F-actin accumulates at the cell surface to promote macropinocytosis and phagocytosis $[15,16]$. Meanwhile, DCs become mature, and substantial actin cytoskeleton rearrangement lead to shut down the endocytosis of soluble antigens and enhance the transportation of vesicles to cell surface $[17,18]$. The internalized vesicles are transported along actin to antigen-processing compartments and are loaded on MHC molecules that promote consecutive surface transport for T-cell activation $[17,19,20]$.

\section{F-actin of DCs and adaptive immune response}

Previous reports have shown that F-actin played at least two crucial roles in immune response. Firstly, actin cytoskeleton rearrangement promotes an immunological synapse formation to organize the distinct supermolecular activation clusters at the contact between DCs and Tcells $[21,22]$. The immunological synapse (IS) is a platform, where various molecules cluster at the interface of T-cells and DCs. The Factin provides the essential support for the platform on the DC side of the synapse [23]. The accumulated F-actin at the IS sustains the stable contact between the DC and the T-cell, which results in T-cell activation $[24,25]$. Conversely, T-cells are not susceptible to activation in case of the presence of a large amount of F-actin filaments that are occupied for the immune response [24]. Secondly, F-actin acts as a scaffold to sustain signalling pathway molecules, which are important for cell-activation and migration [26-28]. In addition, the actin cytoskeleton can modulate the $\mathrm{Ca}^{+2}$ influx by controlling the spatial and temporal distribution of $\mathrm{Ca}^{+2}$ sources and sinks [29-31], which might further influence the activation and maturation of DCs and affect DC-T cell contact and T-cell activation.

\section{Regulators of F-actin in DCs}

The activation and redistribution of F-actin is regulated by calcium signaling, adhesion molecules [32,33] and actin-budling proteins. Fascin-1, an actin-budling protein that regulates F-actin, is solely expressed in mature DCs and is absent in other myeloid cells. Fascin-1 is essential for the formation of IS $[34,35]$. The accumulation of Fascin-1 at the contact of Treg cell-engaged DCs inhibits the synapse formation between DCs and antigen-specific $\mathrm{T}_{\text {conv }}$ cells [25]. Overexpression of Fascin-1 in DCs reverses this inhibition [25].

Immunosuppressors could inhibit the polarization of cytoskeletal proteins in DCs. For example, soluble CD83 (sCD83) is a soluble molecule of the extracellular domain of the membrane-bound CD83 (mCD83), which is a molecular marker for mature DC. sCD83 can induce DCs tolerance and reduce the expression levels of CD40, CD80 and CD83 on DCs to alter their maturation [36]. Increasing evidences have demonstrated that $\mathrm{sCD} 83$ can dramatically change the morphological state of DCs by regulating F-actin organization and the distribution of Fascin-1 on DC [14]. This change may influence the function of DCs. Additionally, interlukin-10 (IL-10) that acts as an immunosuppressor, has been identified as a major cytokine to induce 
immune-tolerance in the immune response. The effects of IL-10 on the biophysical characteristics of DCs have been described to affect the cytoskeleton (F-actin) of mature DCs, and could affect the expression of Fascin 1 and Profilin1, as well as the phosphorylation of the protein Cofilin1 in a concentration-dependent fashion. Moreover, IL-10 made the status of gene transcription and metabolic turnover of mature DCs more active [37].

In addition, the cell division cycle 42 (Cdc42) proteins, a member of the Rho guanosine triphosphatase family, has been shown to regulate the actin organization, cell migration, and proliferation. Cdc42 control of actin dynamics inhibits the secretion of the intracellular molecules to the cell surface and prevents a large amount of invariant chainmajor histocompatibility complex (MHC) class II complexes to express at DCs surface. This retains DCs in an immature state, and cessation of Cdc42 activity during DC maturation facilitates secretion as well as rapid up-regulation of intracellular molecules to the cell surface3. Thus, regulating of the F-actin in DCs could affect the function of DCs. The new DCs regulators, notably the specific regulator for the cytoskeleton of DCs are expected to be investigated in future studies.

\section{The Role of F-actin of DCs in Viral Infection}

DCs play a key role in host defense, linking innate detection of microbes to the activation of pathogen-specific adaptive immune responses [38]. They are considered to be the first line of defense against infection and play a critical role in the regulation of early viral infection. During early events of viral infection, the viral invasion activates natural immune signalling pathway, thereby activating DCs. DCs capture viral particles and present them to $\mathrm{T}$ and $\mathrm{B}$ cells to induce immune responses. Recent reports have confirmed that the infection of virus directly and/or indirectly alters the aggregation of the cytoskeletal proteins, resulting in a beneficial "switch" of the activation of DCs towards viral infection (Figure 1B).

Human Immunodeficiency Virus (HIV) is the most frequently reported virus, which can destroy the cytoskeletal proteins of the immune cells and attack the immune system. HIV recognizes its receptors expressed on DCs, such as DC-specific ICAM-3-grabbing nonintegin (DC-SIGN), mannose receptor (MR) and Langerin. And then, the virions are internalized and are presented to T-cells. Following engagement of DC-SIGN by HIV, a cascade that involves Src kinase proteins, Cdc42, Pak1, and wasp is activated to enhance the formation of actin-rich sheet-like membrane extensions that facilitate HIV transfer to CD4+T cells. Such membrane extensions depend on Cdc42, which regulates actin organization [39].

Although DCs express cell surface receptors for the entry of HIV-1 to the cells, they undergo very limited productive infection as a result of host cell restriction factors. Nevertheless, HIV exploits DCs to infect CD4+T cells rapidly and efficiently via trans-enhancement. This hypothesis was recently supported by in vivo evidence in humanized mice [40]. By localizing to the surface of actin-rich filopodia-like structures of DCs, HIV-1 may increase its likelihood of encountering CD4+T cells [41-43]. However, actin nucleation and an intact cortical actin cytoskeleton are required to maintain HIV-1 association with dendrites and prevent the engulfment of the virus in macropinocytic vesicles [44].

Besides of HIV, there are other kinds of viruses have been reported to affect actin cytoskeleton rearrangements of immune cells in a direct and/or indirect manner. Kaposi's sarcoma-associated herpesvirus
(KSHV) is a $\gamma$-herpesvirus that is highlighted as the causative agent of Kaposi's sarcoma (KS) and primary effusion lymphoma (PEL). Previous studies have shown that KSHV infects fibroblasts via clathrin-mediated endocytosis, and that microtubules are required for the trafficking of viral particles and not for virus internalization $[45,46]$. In addition, KSHV entry induces RhoA GTPase expression and rearrangements of both microtubules and the actin cytoskeleton in fibroblasts [47]. RhoA GTPase is required for viral entry in HEK293 cells [48] and for the association of the viral particles with the microtubules in dermal microvascular cells (DMVEC) [49]. Plasmacytoid DCs (pDCs) are a part of the inflammatory host response cell in KSHV [50], but the role of actin cytoskeleton in DCs for KSHV infection remains unclear. Additionally, Epstein-Barr virus (EBV) enters in DCs by DC-SIGN. However the mechanism of the entry of EBV into DCs and the regulation of movement of DCs by cytoskeletal proteins has not been fully elucidated.

\section{Conclusion}

DC is an important cell that encounters and captures the antigens to provide the first defense of the immune system. Cytoskeletal proteins are essential for the maintenance of the morphology and function of DCs. The regulation of cytoskeletal proteins that are notably found in DCs can affect the function of the cells. Certain viruses can infect DCs by recognizing their receptors. Subsequently, they induce the rearrangement of the actin cytoskeleton and/or bind with cytoskeletal proteins to influence the function of DCs. The investigation of the target of cytoskeletal proteins would be beneficial in suppressing viral infection and dissemination. A potential therapeutic strategy is identified that can intervene with the specific cytoskeletal components of DCs in order to achieve disease treatment.

\section{Acknowledgement}

This work was supported by the grants of the National Nature Science Foundation of China (Grant No. 81601757 and 81500710), and the Innovation Project of Shandong Academy of Medical Sciences.

\section{References}

1. Merad M, Sathe P, Helft J, Miller J, Mortha A (2013) The dendritic cell lineage: ontogeny and function of dendritic cells and their subsets in the steady state and the inflamed setting. Annu Rev Immunol 31: 563-604.

2. Alvarez D, Vollmann EH, von Andrian UH (2008) Mechanisms and consequences of dendritic cell migration. Immunity 29: 325-342.

3. Schulz AM, Stutte S, Hogl S, Luckashenak N, Dudziak D, et al. (2015) Cdc42-dependent actin dynamics controls maturation and secretory activity of dendritic cells. J Cell Biol 211: 553-567.

4. Takenawa T, Miki H (2001) WASP and WAVE family proteins: key molecules for rapid rearrangement of cortical actin filaments and cell movement. J Cell Sci 114: 1801-1809.

5. Chhabra ES, Higgs HN (2007) The many faces of actin: matching assembly factors with cellular structures. Nat Cell Biol 9: 1110-1121.

6. Qualmann B, Kessels MM (2008) Actin nucleation: putting the brakes on Arp2/3. Curr Biol 18: R420-423.

7. Herrmann H, Bar H, Kreplak L, Strelkov SV, Aebi U (2007) Intermediate filaments: from cell architecture to nanomechanics. Nat Rev Mol Cell Biol 8: $562-573$.

8. Fletcher DA, Mullins RD (2011) Cell mechanics and the cytoskeleton. Nature 463: 485-492. 
9. Kobayashi M, Azuma E, Ido M, Hirayama M, Jiang Q, et al. (2001) A pivotal role of Rho GTPase in the regulation of morphology and function of dendritic cells. J Immunol 167: 3585-3591.

10. Geli MI, Riezman H (1998) Endocytic internalization in yeast and animal cells: similar and different. J Cell Sci 111: 1031-1037.

11. Wickstead B, Gull K (2011) The evolution of the cytoskeleton. J Cell Biol 194: 513-525.

12. Miermans CA, Kusters RP, Hoogenraad CC, Storm C (2017) Biophysical model of the role of actin remodeling on dendritic spine morphology. PLoS One 12: e0170113.

13. Koganezawa N, Hanamura K, Sekino Y, Shirao T (2017) The role of drebrin in dendritic spines. Mol Cell Neurosci 7431: 30205-30206.

14. Kotzor N, Lechmann M, Zinser E, Steinkasserer A (2004) The soluble form of CD83 dramatically changes the cytoskeleton of dendritic cells. Immunobiology 209: 129-140.

15. Niedergang F, Chavrier P (2004) Signaling and membrane dynamics during phagocytosis: many roads lead to the phagos(R)ome. Curr Opin Cell Biol 16: 422-428.

16. Kerr MC, Teasdale RD (2009) Defining macropinocytosis. Traffic 10: 364-371.

17. West MA, Wallin RP, Matthews SP, Svensson HG, Zaru R, et al. (2004) Enhanced dendritic cell antigen capture via toll-like receptor-induced actin remodeling. Science 305: 1153-1157.

18. Garrett WS, Chen LM, Kroschewski R, Ebersold M, Turley S, et al. (2000) Developmental control of endocytosis in dendritic cells by Cdc42. Cell 102: 325-334.

19. Trombetta ES, Mellman I (2005) Cell biology of antigen processing in vitro and in vivo. Annu Rev Immunol 23: 975-1028.

20. Kaksonen M, Toret CP, Drubin DG (2006) Harnessing actin dynamics for clathrin-mediated endocytosis. Nat Rev Mol Cell Biol 7: 404-414.

21. Grakoui A, Bromley SK, Sumen C, Davis MM, Shaw AS, et al. (1999) The immunological synapse: a molecular machine controlling $\mathrm{T}$ cell activation. Science 285: 221-227.

22. Monks CR, Freiberg BA, Kupfer H, Sciaky N, Kupfer A (1998) Threedimensional segregation of supramolecular activation clusters in $\mathrm{T}$ cells. Nature 395: 82-86.

23. Al-Alwan MM, Liwski RS, Haeryfar SM, Baldridge WH, Hoskin DW, et al. (2003) Cutting edge: dendritic cell actin cytoskeletal polarization during immunological synapse formation is highly antigen-dependent. J Immunol 171: 4479-4483.

24. Lin W, Suo Y, Deng Y, Fan Z, Zheng Y, et al. (2015) Morphological change of CD4(+) T cell during contact with DC modulates T-cell activation by accumulation of F-actin in the immunology synapse. BMC Immunol 16: 49.

25. Chen J, Ganguly A, Mucsi AD, Meng J, Yan J, et al. (2017) Strong adhesion by regulatory $\mathrm{T}$ cells induces dendritic cell cytoskeletal polarization and contact-dependent lethargy. J Exp Med 214: 327-338.

26. Bubeck Wardenburg J, Pappu R, Bu JY, Mayer B, Chernoff J, et al. (1998) Regulation of PAK activation and the $\mathrm{T}$ cell cytoskeleton by the linker protein SLP-76. Immunity 9: 607-616.

27. Villalba M, Coudronniere N, Deckert M, Teixeiro E, Mas P, et al. (2000) A novel functional interaction between Vav and PKCtheta is required for TCR-induced T cell activation. Immunity 12: 151-160.

28. Babich A, Li S, O'Connor RS, Milone MC, Freedman BD, et al. (2012) Factin polymerization and retrograde flow drive sustained PLCgammal signaling during $T$ cell activation. J Cell Biol 197: 775-787.

29. Quintana A, Schwindling C, Wenning AS, Becherer U, Rettig J, et al. (2007) $\mathrm{T}$ cell activation requires mitochondrial translocation to the immunological synapse. Proc Natl Acad Sci U S A 104: 14418-14423.

30. Nolz JC, Gomez TS, Zhu P, Li S, Medeiros RB, et al. (2006) The WAVE2 complex regulates actin cytoskeletal reorganization and CRAC-mediated calcium entry during T cell activation. Curr Biol 16: 24-34.

31. Quintana A, Schwarz EC, Schwindling C, Lipp P, Kaestner L (2006) Sustained activity of calcium release-activated calcium channels requires translocation of mitochondria to the plasma membrane. J Biol Chem 281: 40302-40309.

32. Lek HS, Morrison VL, Conneely M, Campbell PA, McGloin D, et al. (2013) The spontaneously adhesive leukocyte function-associated antigen-1 (LFA-1) integrin in effector T cells mediates rapid actin- and calmodulin-dependent adhesion strengthening to ligand under shear flow. J Biol Chem 288, 14698-14708.

33. Schell MJ, Irvine RF (2006) Calcium-triggered exit of F-actin and IP(3) 3 kinase A from dendritic spines is rapid and reversible. Eur J Neurosci 24: 2491-2503.

34. Al-Alwan MM, Rowden G, Lee TD, West KA (2001) Fascin is involved in the antigen presentation activity of mature dendritic cells. J Immunol 166: 338-345.

35. Yamakita Y, Matsumura F, Lipscomb MW, Chou PC, Werlen G, et al. (2011) Fascin1 promotes cell migration of mature dendritic cells. J Immunol 186: 2850-2859.

36. Yang Y, Xin Z, Chu J, Li N, Sun T (2014) Involvement of Caveolin-1 in CD83 Internalization in Mouse Dendritic Cells. Cell Transplant 24: 1395-1404.

37. $\mathrm{Xu} \mathrm{X}$, Liu X, Long J, Hu Z, Zheng Q, et al. (2017) Interleukin-10 reorganizes the cytoskeleton of mature dendritic cells leading to their impaired biophysical properties and motilities. PLoS One 12: e0172523.

38. Banchereau J, Briere F, Caux C, Davoust J, Lebecque S, et al. (2000) Immunobiology of dendritic cells. Annu Rev Immunol 18: 767-811.

39. Nikolic DS, Lehmann M, Felts R, Garcia E, Blanchet FB, et al. (2011) HIV-1 activates Cdc42 and induces membrane extensions in immature dendritic cells to facilitate cell-to-cell virus propagation. Blood 118: 4841-4852.

40. Sewald X, Ladinsky MS, Uchil PD, Beloor J, Pi R, et al. (2015) Retroviruses use CD169-mediated trans-infection of permissive lymphocytes to establish infection. Science 350: 563-567.

41. Cavrois M, Neidleman J, Greene WC (2008) The achilles heel of the trojan horse model of HIV-1 trans-infection. PLoS Pathog 4: e1000051.

42. Do T, Murphy G, Earl LA, Del Prete GQ, Grandinetti G, et al. (2014) Three-dimensional imaging of HIV-1 virological synapses reveals membrane architectures involved in virus transmission. J Virol 88: 10327-10339.

43. Yu HJ, Reuter MA, McDonald D (2008) HIV traffics through a specialized, surface-accessible intracellular compartment during transinfection of T cells by mature dendritic cells. PLoS Pathog 4: e1000134.

44. Donahue DA, Schwartz O (2016) Actin' on HIV: How Dendritic Cells Spread Infection. Cell Host Microbe 19: 267-269.

45. Akula SM, Naranatt PP, Walia NS, Wang FZ, Fegley B, et al. (2003) Kaposi's sarcoma-associated herpesvirus (human herpesvirus 8) infection of human fibroblast cells occurs through endocytosis. J Virol 77: 7978-7990.

46. Naranatt PP, Krishnan HH, Smith MS, Chandran B (2005) Kaposi's sarcoma-associated herpesvirus modulates microtubule dynamics via RhoA-GTP-diaphanous 2 signaling and utilizes the dynein motors to deliver its DNA to the nucleus. J Virol 79: 1191-1206.

47. Sharma-Walia N, Naranatt PP, Krishnan HH, Zeng L, Chandran B (2004) Kaposi's sarcoma-associated herpesvirus/human herpesvirus 8 envelope glycoprotein $\mathrm{gB}$ induces the integrin-dependent focal adhesion kinaseSrc-phosphatidylinositol 3-kinase-rho GTPase signal pathways and cytoskeletal rearrangements. J Virol 78: 4207-4223.

48. Veettil MV, Sharma-Walia N, Sadagopan S, Raghu H, Sivakumar R, et al. (2006) RhoA-GTPase facilitates entry of Kaposi's sarcoma-associated herpesvirus into adherent target cells in a Src-dependent manner. J Virol 80: 11432-11446.

49. Raghu H, Sharma-Walia N, Veettil MV, Sadagopan S, Caballero A, et al. (2007) Lipid rafts of primary endothelial cells are essential for Kaposi's sarcoma-associated herpesvirus/human herpesvirus 8-induced phosphatidylinositol 3-kinase and RhoA-GTPases critical for microtubule dynamics and nuclear delivery of viral DNA but dispensable for binding and entry. J Virol 81: 7941-7959. 
Citation: Lin W, Wang X (2017) Regulating DCs in Innate Immune Response and Infection by Cytoskeletal Proteins. J Immuno Biol 2: 124. doi: $10.4172 / 2476-1966.1000124$

Page 5 of 5

50. Karouni M, Kurban M, Abbas O (2016) Plasmacytoid dendritic cells in skin lesions of classic Kaposi's sarcoma. Arch Dermatol Res 308: 487-492.

This article was originally published in a special issue, entitled:

"Immunoregulation of Innate Immune Cells in the Disease", Edited by Wei

Lin, Department of microbiology, Shandong Academy of Medical Sciences,

People's Republic of China 